\title{
Natural products synthesis: palytoxin
}

\author{
Yoshito Kishi \\ Department of Chemistry, Harvard University, Cambridge, MA 02138, U.S.A.
}

\begin{abstract}
The first part of this presentation is concerned with conformational analysis of $C$ and $O$-saccharides. A useful model for predicting the solution conformation of these classes of compounds is presented. The second part is concerned with the total synthesis of palytoxin carboxylic acid with focus on the bond formation at the C.7-C.8, C.51-C.52, and C.75-C.76 positions.
\end{abstract}

Palytoxin, the toxic principle isolated from marine soft corals of the genus Palythoa, is the most poisonous substance known to date except for a few naturally occurring proteins found in bacteria and plants (ref. 1). The gross structure of palytoxin was elucidated in 1981 by two groups independently, the one led by Professor Hirata at Nagoya in Japan (ref. 2a) and the other by Professor Moore at Honolulu in the United States (ref. 2b). Thus, it is evident that palytoxin is uniquely distinct from molecules, with which organic chemists have previously dealt, in terms of magnitude of molecular size and of structural complexity. Shortly after the gross structure became available, we decided to undertake investigations on this extraordinary natural product; our interests were primarily two-fold, chemical synthesis and conformational analysis. However, we realized that the information given by the gross structure was not sufficient to address our questions properly. For this reason, the very first step of this project was to establish unambiguously the complete structure of palytoxin, and we decided to depend principally on organic synthesis to do so. On the basis of extensive efforts approximately for two years, we succeeded in elucidating the complete structure of palytoxin in 1982 (ref. 3).

In this presentation, we would like to review our efforts on the chemical synthesis and conformational analysis of palytoxin.

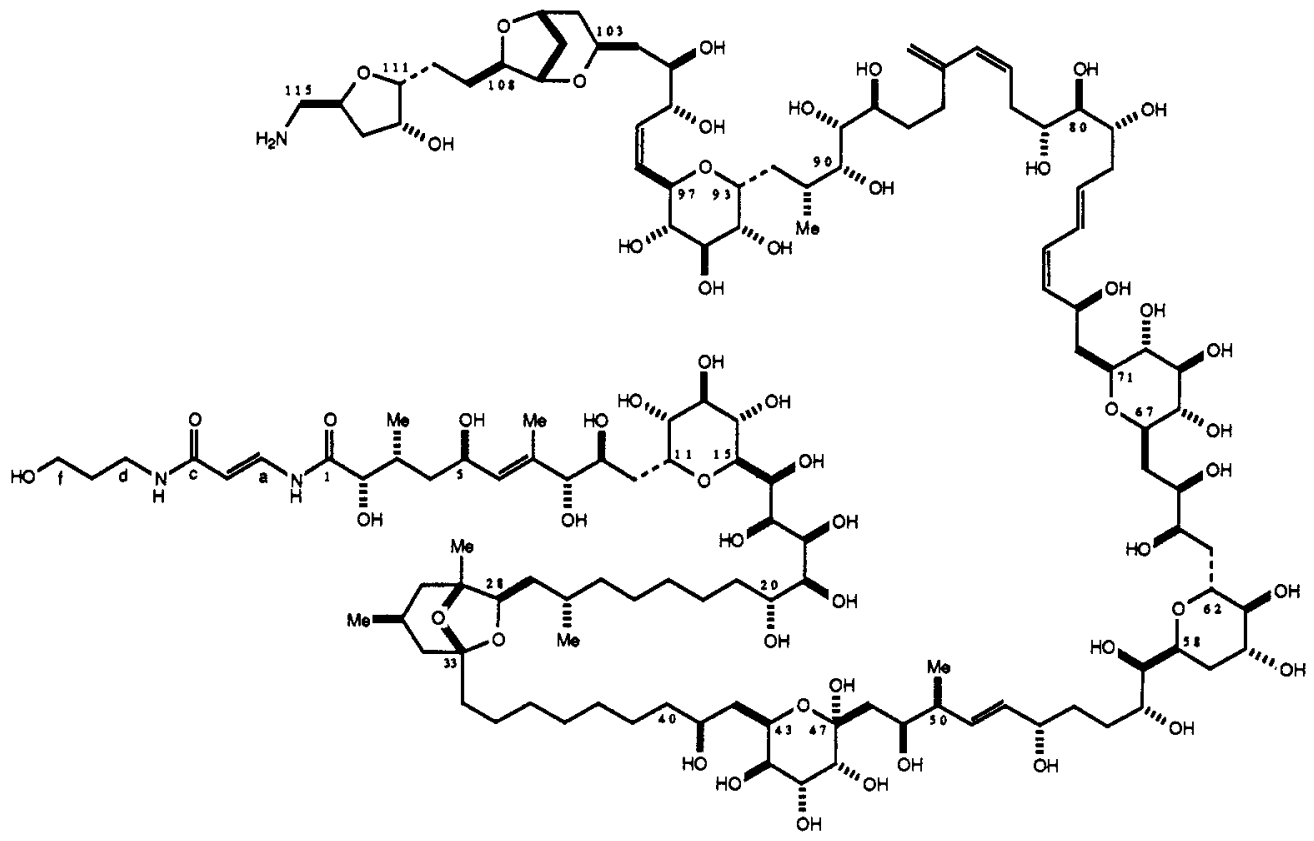




\section{CONFORMATIONAL ANALYSIS}

Since major parts of the palytoxin structure could be viewed as $C$-oligosaccharides, we became interested in comparing the conformational preference of glycosides to that of corresponding $C$. glycosides (ref. 4). Regarding the $O-R$ bond at the anomeric center, $\alpha$ (axial)-glycosides are known to prefer the conformer $1 \mathrm{~A}$ over the conformers $1 \mathrm{~B}$ and $1 \mathrm{C}$ due to (1) steric destabilization of $1 \mathrm{C}$ over $\mathbf{1 A}$ and 1B, (2) stereoelectronic stabilization of $\mathbf{1 A}$ (and 1C) over $1 \mathrm{~B}$ - referred to as the exo-anomeric effect (ref. 5), and (3) steric destabilization of $1 \mathbf{B}$ over $1 \mathbf{A}$. Thus, the similarity or dissimilarity of the conformational behavior between parent glycosides and corresponding $C$-glycosides depends primarily upon the degree of stabilization due to the stereoelectronic effect - compare $1 \mathrm{~A}-\mathrm{C}$ with $2 \mathrm{~A}-\mathrm{C}$. To the best of our knowledge, however, there was no rigorous experimental data available to estimate the extent of the stabilization due to the exo-anomeric effect.

For this reason, a number of $C$-glycosides were synthesized and subjected to ${ }^{1} \mathrm{H}-\mathrm{NMR}$ studies. The

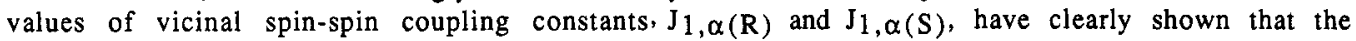
preferred conformation around the C.1-C. $\alpha$ bond does correspond to $2 \mathrm{~A}$. The observed values of $\mathrm{J}_{1, \alpha}(\mathrm{S})=10 \sim 12 \mathrm{~Hz}$ and $\mathrm{J}_{1, \alpha(R)}=3 \sim 4 \mathrm{~Hz}$ could be translated into a dihedral angle by using the Karplus equation, yielding approximately $55^{\circ}$ for the O.1-C.1-C. $\alpha-C . \beta$ torsional angle. This value is remarkably close to the corresponding torsional angle $\left(55 \pm 5^{\circ}\right.$ in solution; $63^{\circ}$ in crystal) of methyl $\alpha$ -

glucopyranoside.

Encouraged by these results, we examined the conformational preference of $\beta$ (equatorial)- $C$ glycosides as well. Based on a similar analysis, we anticipated the conformer $\mathbf{4 A}$ to be preferred over the conformers $4 \mathrm{~B}$ and $4 \mathrm{C}$. As before, the $1 \mathrm{H}-\mathrm{NMR}$ data of $\beta-C$-glycosides was found to be beautifully consistent with this expectation.

The ${ }^{1}$ H-NMR studies outlined have clearly established that $\alpha$ (axial)- and $\beta$ (equatorial)- $C$-glucosides exist predominantly in the conformation of $2 \mathrm{~A}$ and $4 \mathrm{~A}$, respectively. Like the corresponding glycoside, the $C$-glycoside is probably not conformationally rigid. Nonetheless, the weighted average of available conformers corresponds extremely well to the one predicted solely on the basis of the consideration of steric interactions. Thus, although our results do not preclude the existence of a stereoelectronic stabilization, the exo-anomeric effect is not the major factor why glycosides adopt preferentially the conformation $1 \mathrm{~A}$ or $3 \mathrm{~A}$.

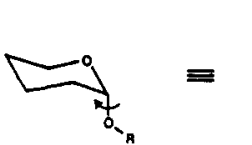

1

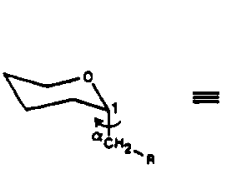

2

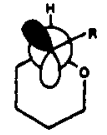

A

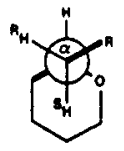

A

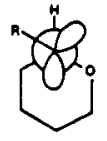

B

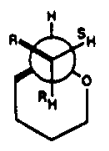

B

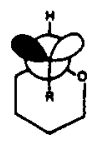

C

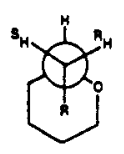

C

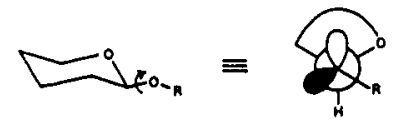

A

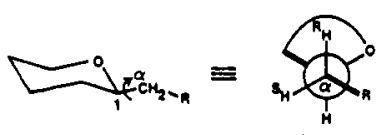

4
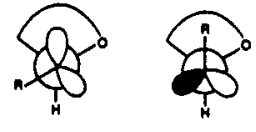

B

C

Naturally, we were interested in extending this study to 1,2-, 1,3-, 1,4-, and 1,6-C-disaccharides. For this purpose, we needed to develop synthetic methods for this class of compounds. Fortunately, our experience with the palytoxin synthesis was of great help in solving the problem. A number of $C$ disaccharides, including $C$-maltose and $C$-cellobiose, were synthesized and subjected to ${ }^{1} \mathrm{H}-\mathrm{NMR}$ studies.

Let us use methyl $C$-maltoside as an example. From the spin-spin coupling constants between the C.1' and C. $\alpha$ protons, it is evident that the $C$-glycosidic bond adopts preferentially the predicted conformation. However, the analysis of the conformational preference around the non- $C$-glycosidic C.4-C. $\alpha$ bond is somewhat more complicated. We have found it is convenient to use a diamond lattice for the analysis. Among the three ideal staggered conformers A-C available for this case, conformer $C$ seems to suffer from the most severe steric interactions since the C. $\alpha-C .1^{\prime}$ bond is surrounded by the C.4-C.3 and C.4-C.5 bonds. 1,3-Diaxial-like interactions are obvious in both conformers $\mathbf{A}$ and $\mathbf{B}$. However, inspection of the drawing on a diamond lattice may suggest that conformer $\mathbf{A}$ is slightly less sterically crowded than conformer $\mathbf{B}$; there is one 1,3-diaxial-like interaction in conformer $\mathbf{A}$ (C.1'C. $\alpha / C .3-O H)$ whereas there are two 1,3-diaxial-like interactions in conformer B (C.1'-C. $\alpha / C .5-C .6$ and C.1'-O/C.4-C.5).

The ${ }^{1}$ H-NMR studies have demonstrated that methyl maltoside does not exists in either of the ideal staggered conformers $\mathbf{A}$ or $\mathbf{B}$ but exists as a mixture of the conformers $\mathbf{A}$ and $\mathbf{B}$, weighted towards the former, or in a conformer slightly deviated from $\mathbf{A}$. The conformational behavior of this glycoside seems to agree well with that of the corresponding parent disaccharide. 
It is noteworthy that the conformational preference around the $C$-glycosidic bond is so overwhelming that a structural deviation from the ideal staggered conformer to avoid the steric interactions takes place in rotating principally the non-glycosidic C.4-C. $\alpha$ bond rather than the glycosidic C.1'-C. $\alpha$ bond. It is interesting that this phenomenon has been observed also on the parent glycosides (ref. 5). The recognition of this phenomenon allows to predict the conformational behavior of di- and higher- $C$-saccharides and their parent substances and, more importantly, to suggest the structural modification needed to change the conformational preference of given glycosides. For example, the most serious steric destabilization in the conformer $A$ is due to a 1,3diaxial-like interaction between the C.3 hydroxy group and C.1'-C. $\alpha$ bond. Therefore, a significant effect on the conformational preference of this compound should be observed on removing the C. 3 hydroxy group or changing its configuration. Indeed, the ${ }^{1} \mathrm{H}-\mathrm{NMR}$ studies of methyl C.3-deoxy-Cmaltoside have proved this prediction to be true.

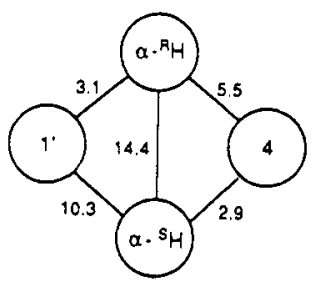

spin-spin coupling

diagram for $\mathrm{X}=\mathrm{OH}$

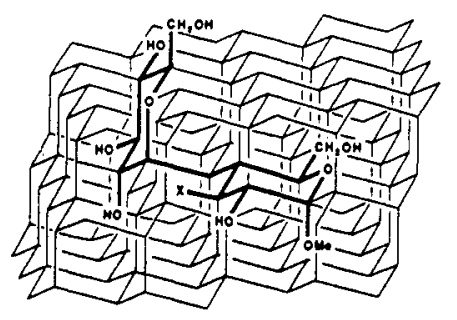

A<smiles>[X]C1[C@H](O)[C@@H](OC)O[C@H](CO)[C@@H]1C[C@H]1OC(CO)(CO)[C@@H](O)[C@H]1O</smiles>

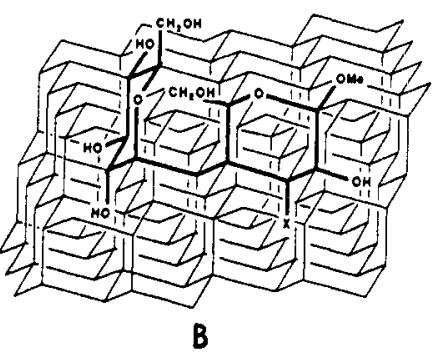

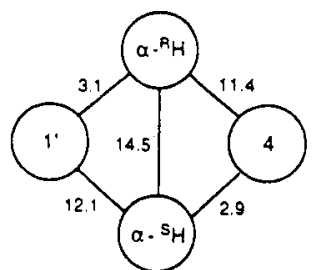

spin-spin coupling

diagram for $X=H$

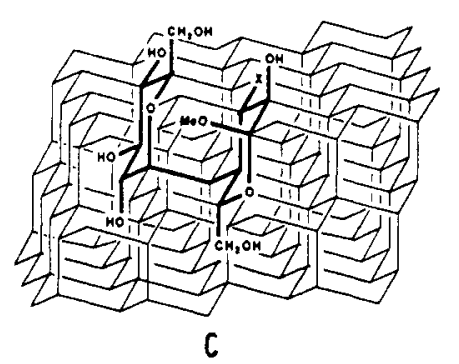

Conformational analysis of methyl $C$-cellobioside was possible in the same manner; in this case, the C.5 hydroxymethyl group causes a 1,3-diaxial-like interaction, the structural modification of which resulted in a dramatic effect on the conformational preference.

We were interested in extending the predictive value of this model to the corresponding parent glycosides. Needless to point out, the conformational analysis of oligosaccarides is an active research subject, and a variety of methods, including nuclear Overhauser effects (NOE), $\mathrm{T}_{1}$ measurements and force-field calculation, have extensively been utilized (ref. 6). However, we must emphasize that we are not attempting to use these methods as the principal means of conformational analysis.

Experimentally, based on the values of vicinal spin-spin coupling constants, we first establish the conformational preference of the carbon series and second compare the conformational behavior between $O$ - and $C$-disaccharides through NOE and $T_{1}$ data. This approach has recently been applied to several disaccharides in our laboratories (ref. 7).

Let us use the case of methyl C.3-deoxymaltoside and its carbon analogue for demonstration. Diamond lattice analysis of both compounds suggests that the preferred conformation for each should be the one depicted on the top right corner. The vicinal spin-spin coupling constants in the carbon series firmly support this prediction. In this conformation, $H_{.} 1^{\prime}$ and $H .3(e q)$ are in close enough proximity that NOE enhancements should be observable. Indeed, the experimental results supported this analysis.<smiles>CO[C@H]1O[C@H](CO)[C@@H](C[C@@H]2O[C](CO)[C@@H](O)[C@H](O)[C@H]2O)C[C@H]1O</smiles>

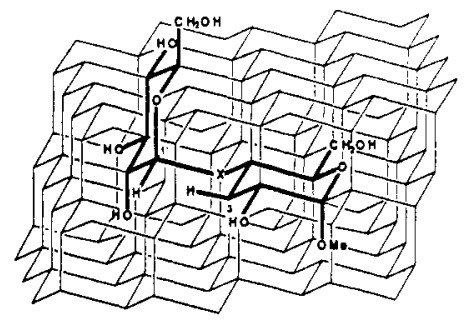

$T_{1}$ Values (sec)

$\begin{array}{llll}\text { Protons } & \mathrm{X}=\mathrm{O} & \mathrm{X}=\mathrm{CH}_{2} & \mathrm{X}=\mathrm{CD}_{2} \\ \text { H.1' } & 0.94 & 0.72 & 0.71 \\ \text { H.3(eq) } & 0.36 & 0.36 & 0.37 \\ \text { H.4 } & 0.79 & 0.36 & 0.81\end{array}$

NOE (\%)

$\begin{array}{llll}\text { Protons } & \mathrm{X}=\mathrm{O} & \mathrm{X}=\mathrm{CH}_{2} & \mathrm{X}=\mathrm{CD}_{2} \\ \text { H.1' } \rightarrow \text { H.3(eq) } & 6.9 & 5.1 & 5.2 \\ \text { H.1' } \rightarrow \text { H.4 } & 7.2 & 0.0 & 5.1 \\ \text { H.3 }(\mathrm{eq}) \rightarrow \text { H.1' } & 8.7 & 6.1 & 9.1\end{array}$


However, on irradiation of H.1', a distinct NOE was observed on H.4 of the parent glycoside whereas no NOE was detected on $\mathrm{H} .4$ of the corresponding $C$-disaccharide. Since the $\mathrm{CH}_{2}$-bridge of the carbon analogue was the likely source of this discrepancy, the corresponding bisdeuterated compound was prepared. Indeed, the relaxation time for $\mathrm{H} .4$ of bisdueterated derivative was much longer than that observed for the non-deuterated substance, reflecting the decrease of spin-spin interactions between $\mathrm{H} .4$ and the $\mathrm{CD}_{2}$-bridge. Irradiation of $\mathrm{H} . \mathrm{l}^{\prime}$ produced a $5.1 \%$ enhancement of $\mathrm{H.4}$ of this substance. Thus, the absence of an NOE between H.1' and H.4 in the carbon analogue is primarily due to the spinspin interactions of $\mathrm{H.4}$ with the bridging methylene protons rather than to conformational dissimilarity between the $O$ - and $C$-disaccharides.

The observation of an NOE between H.1' and H.4 in these compounds was not expected for the ideal conformation shown. Consideration of this result, in conjunction with the appropriate vicinal spinspin coupling constants for the carbon analogue, brings to light at least two plausible explanations; the system may be twisted slightly away from the ideal staggered arrangement around the nonglycosidic bond or a small amount of another conformer may be present in the solution. Further study is necessary to clarify the exact nature of this effect.

In summary, we have shown that $C$-disaccharides and the corresponding parent disaccharides are conformationally similar to each other. In addition, we have demonstrated that analysis of steric interactions primarily around the non-glycosidic bond in the $C$-and $O$-disaccharides using a diamond lattice provides a useful model for predicting the preferred conformation in these systems. In all the cases examined, the experimentally-determined preferred conformation was very close, although not identical, to the predicted conformation. It is our belief that this approach will have an interesting impact on conformational analysis of carbohydrates and related substances.

Back to the palytoxin project, the ${ }^{1} \mathrm{H}-\mathrm{NMR}$ studies have convincingly demonstrated the preferred local conformation; for example, the C.86-C.110 region is the one depicted below. We may emphasize the fact that the availability of non-deuterated as well as specifically-deuterated synthetic intermediates made the $1_{\mathrm{H}-\mathrm{NMR}}$ analysis simple and conclusive. Applying the same technique, we can reach a conclusion on the preferred conformation of palytoxin itself in aqueous methanol. Although we are confident of this conclusion, one serious question should be addressed about this approach. Namely, the conclusion obtained through this study could be accompanied by small errors in determining the local preferred conformation, which might result in a serious error in a global sense. In order to avoid this potential error, we have been engaged in the development of structurally-stiff chemical rulers, resulting in one such system, composed of organic solvent soluble oligopeptides with 310 -helical structure (ref. 8). We hope that this will help to determine the global solution-conformation of palytoxin.
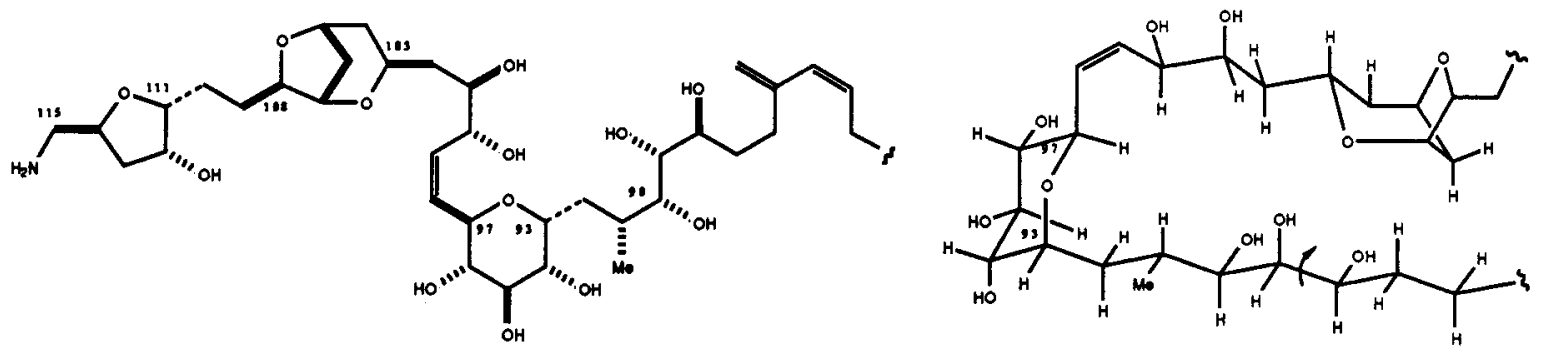

\section{SYNTHESIS}

By the summer of 1985 , we had developed the syntheses of the eight key building blocks. Each synthesis has been improved and polished up to the level satisfactory in terms of overall efficiency and practicability. For example, before the current route developed, the C.8-C.22 segment had been synthesized by four different routes. Each of the syntheses had provided numerous opportunities to discover exciting and intriguing chemistry, which is, in my view, worthy in its own right. However, we may emphasize the fact that the progress beyond this stage critically depended on the availability of these building blocks. In this connection, we should point out that each of these building blocks are available in multi-gram quantities with relatively small efforts (ref. 9, 10, 11).

Having established the practical synthetic route to all the key building blocks, we were now ready to address the question of how we could couple them together. Some of the couplings could be carried out relatively straightforwardly; for example, the C.22-C.23 and C.37-C.38 bonds were formed via Wittig reaction, followed by hydrogenation. However, some other couplings turned out to be much more challenging. One of these was the bond-formation at the C.7-C.8 position. Using suitable model systems, we have evaluated the feasibility and applicability of various bond-forming reactions, and found the $\mathrm{Ni}(\mathrm{II}) / \mathrm{Cr}(\mathrm{II})$-mediated coupling reaction to be the best by far for this purpose. Let us briefly review how this coupling reaction was developed through the palytoxin project. 
In connection with the synthesis of C.8-C.22 segment, we were faced with the task of transforming the aldehyde or its synthetic equivalents into the allylic alcohol, which seemed possible through routine synthetic operations. However, we soon realized that standard synthetic routes such as Wittig and aldol approaches were not as practical as we had hoped. Among the many possibilities attempted, a coupling reaction utilizing organocuprates gave, at least in the model series, very promising results. However, in spite of extensive efforts, we were unable to generate the desired organocuprate reagent from the trans-iodoolefin.

The clue to the solution came from the timely work of Professor Nozaki and co-workers on the chromium(II)-mediated addition of alkenyl halides to aldehydes (ref. 12). After much trial-and-error experimentation, we were able to accomplish the required coupling by adding $\mathrm{CrCl}_{2}$ to a $\mathrm{DMSO}$ solution of aldehyde and iodoolefin at room temperature in the absence of oxygen. This reaction warrants several additional comments. First, we have examined a large number of highly-oxygenated substrates, and found the coupling to be remarkably effective even for polyfunctional cases, including $\alpha$-oxygenated aldehydes with iodoolefins or $\beta$-iodoenones. Second, the stereochemistry of trans- as well as cis-iodoolefins is retained at least in the cases of disubstituted iodoolefins and trisubstituted trans-iodoolefins. Third, with respect to the newly introduced chiral center, this process produces a mixture of two possible diastereomers with a moderate-to-good preference of one stereoisomer. It is worth mentioning that the major products produced from $\alpha$-alkoxy and $\alpha, \beta$ bisalkoxy aldehydes have the stereochemistry opposite to cuprate or Grignard products.<smiles>CC1(C)OC[C@@H](CC2O[C@H](C=O)[C@@H]([18OH])[C@H](O)[C@H]2[OH2+])O1</smiles>

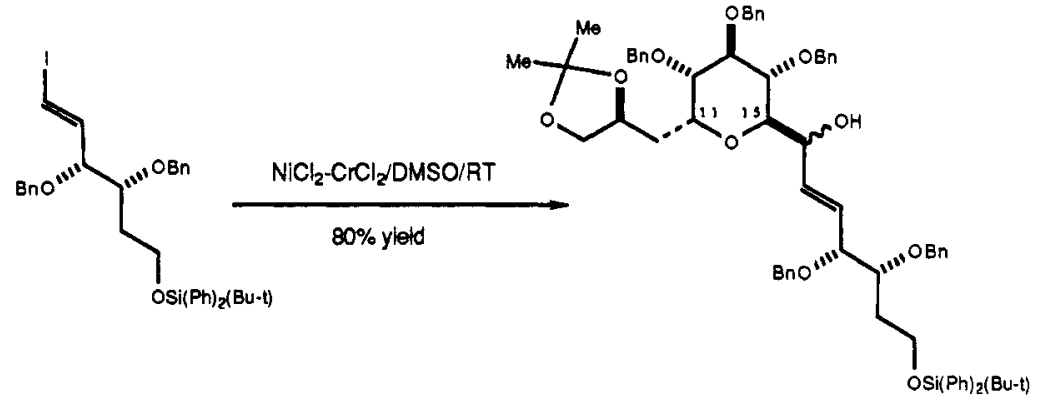

The $\mathrm{Cr}$ (II)-mediated coupling reaction provided an excellent solution to our problem except for one technical difficulty we had yet to overcome. Unlike the $\mathrm{Cr}(\mathrm{II})$-mediated coupling of allyl halides with aldehydes (ref. 13), the success of this coupling mysteriously depended on the source and batch of $\mathrm{CrCl}_{2}$. We tested also the home-made $\mathrm{Cr}$ (II)-reagent without success. These facts naturally suggested an intriguing possibility that the success of this coupling might depend on some unknown contaminant in $\mathrm{CrCl}_{2}$. For this reason, we have examined the effect of transition metals for the $\mathrm{Cr}(\mathrm{II})$-mediated coupling reaction and found that $\mathrm{NiCl}_{2}$, when added to the reaction medium, has a dramatic effect. Although we have not yet established whether the effectiveness of the commercially available $\mathrm{CrCl}_{2}$ samples is due to $\mathrm{Ni}$ salt or some other metal contaminant, it is now possible to achieve the coupling using $\mathrm{CrCl}_{2}$ from any source with excellent reproducibility. It is important to keep the $\mathrm{NiCl}_{2}$ content in $\mathrm{CrCl}_{2}$ low [about $0.1 \% \sim 1 \%(\mathrm{w} / \mathrm{w})$ ] to avoid formation of dienes from iodoolefins due to homocoupling. Reactions are fast in DMF, DMF-THF, or DMF-(Me) $2 S$, which is usually the choice of solvent for coupling of iodoenones. Reactions are slower in DMSO but this often gives much cleaner results for the coupling of iodoolefins.

With respect to the possible mechanism for the activation of an alkenyl iodide, this process may involve initial reduction of $\mathrm{Ni}(\mathrm{II})$ into $\mathrm{Ni}(\mathrm{I})$ or $\mathrm{Ni}(\mathrm{O})$ by $\mathrm{Cr}(\mathrm{II})$, oxidative addition of an alkenyl iodide to $\mathrm{Ni}(\mathrm{I})$ or $\mathrm{Ni}(0)$, and then transmetallation with $\mathrm{Cr}(\mathrm{II})$ or $\mathrm{Cr}(\mathrm{III})$, to generate the organometallic reagent which then couples with an aldehyde. On the other hand, Ni(II) is regenerated and completes the cycle.

There was one additional technical difficulty we had to overcome for the successful application of this reaction to the C.7-C.8 bond formation. Namely, it was important to use a large excess of vinyliodide for achieving the $\mathrm{Ni}(\mathrm{II}) / \mathrm{Cr}$ (II)-mediated coupling reaction efficiently, which was not a serious problem as long as the vinyliodides were inexpensive. Unfortunately, the vinyliodide required for the C.7-C.8 bond formation was not cheap by any means, which forced us to search for a method to increase the efficiency of the catalytic cycle. For this reason, we studied the effect of C.5 protecting group on the coupling reaction using a model system. The results were dramatic; with a silyl protecting group at the C.5 position, the coupling reaction could be achieved in excellent yield even with 2 equivalents of the vinyliodide. Although the reason(s) for the dramatic improvement of efficiency of the catalytic cycle still remained unclear, this observation, i.e. a high efficiency on the substrate with a silyl protecting group(s), is quite general. We have found one additional bonus from this model study. The C.8stereoselectivity was vastly improved with the $t$-butyldimethyl silyl (TBS) protecting group. 
The $\mathrm{Cr}(\mathrm{II})$-mediated coupling allows a carbon-carbon bond formation between alkenyl halides and aldehydes, which can usually be achieved by traditional organometallic reagents such as Grignard, lithium, or cuprate. However, there are several unique characteristics of this reaction.

Experimentally, activation of a carbon-iodine bond in the presence of an aldehyde offers an attractive convenience for many cases. In our view, this coupling reaction has demonstrated its potential and uniqueness for polyfunctional substrates, for which conventional organometallic reagents are difficult to apply. The coupling reaction of the C.1-C.7 segment with the C.8-C.51 segment best illustrates this point; the $\mathrm{Ni}(\mathrm{II}) / \mathrm{Cr}(\mathrm{II})$-mediated coupling reaction under the standard conditions using 2 equivalents of the vinyliodide yielded the expected product in $75 \%$ yield as a $5: 1$ mixture of the C.8 diastereomers favoring the desired product. The minor undesired stereoisomer was converted into the desired stereoisomer via an oxidation-reduction process. After acetylation, hydrolysis of the acetonide group under mild acidic conditions furnished the complete left half of palytoxin (ref. 14).

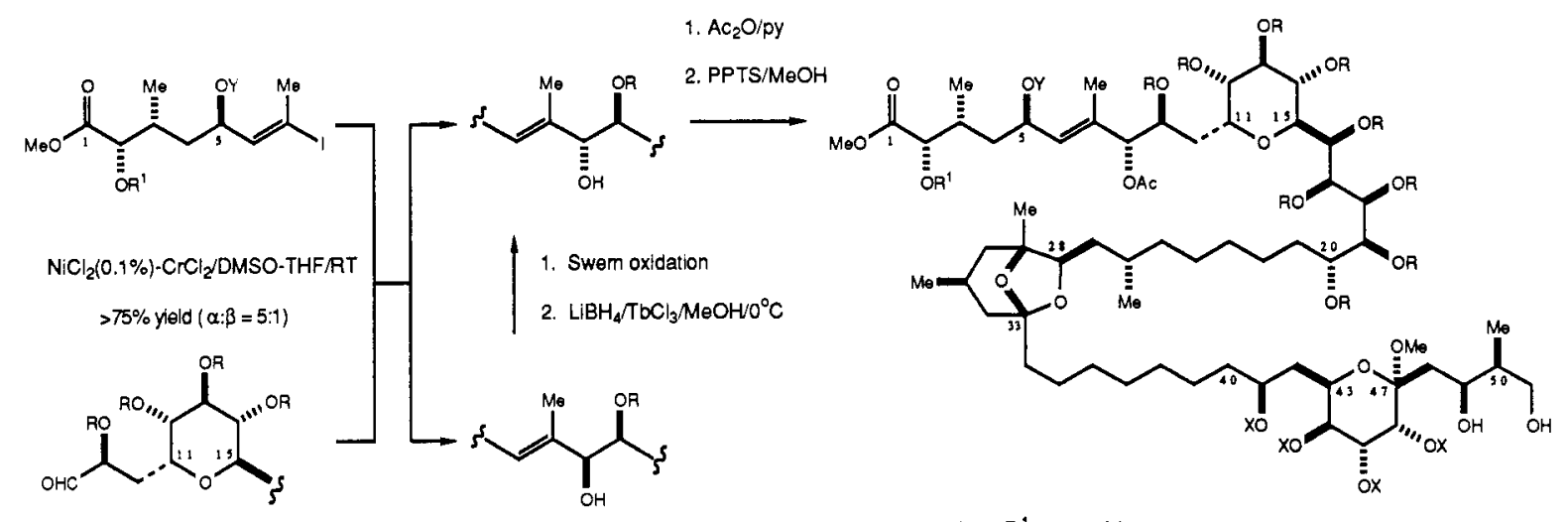

$\mathrm{R}=4-\mathrm{MeOPhCH}_{2}, \mathrm{R}^{1}=2,4-(\mathrm{MeO})_{2} \mathrm{PhCH}_{2}, \mathrm{X}=\mathrm{Bz}, \mathrm{Y}=(\mathrm{t}-\mathrm{Bu})(\mathrm{Me})_{2} \mathrm{Si}$

Let us briefly review the reasons for our choice of the protecting groups:

1. The C.5 protecting group was chosen because of the maximum efficiency of the couping reaction.

2. The C.1 protecting group was chosen primarily because of the convenience of preparation. We should point out the fact that attempted deprotection of this group under basic conditions met with some difficulties. However, this problem was nicely solved by deprotecting the C.5 silyl group first, resulting in the $\delta$-lactone which was smoothly hydrolyzed on brief treatment with an aqueous base or acid.

3. The choice of $p$-methoxyphenylmethyl (MPM) was made because of its stability under the variety of reaction conditions used for the synthesis of this segment and also because of the ease of deprotection under the conditions developed by the late Dr. Oikawa (ref. 15). However, the technical difficulty encountered here was the overall efficiency; there are ten MPM groups, counting the $o, p$-dimethoxyphenylmethyl (DMP) group at the C.2 positions, which means the overall efficiency of the deprotection is only $60 \%$ even when it is achieved in $95 \%$ efficiency per group. This problem was solved by extensive experimentation, defining the suitable conditions to achieve the deprotection in higher than $95 \%$ overall yield.

4. During the DDQ deprotection study, we noticed that the rate of deprotection of the C.2 MPM group was considerably slower than the remaining MPM groups. Changing the electronic nature of this group, i.e. DMP, provided the enhancement in the rate of deprotection, comparable with the rate of deprotection of the remaining MPM groups.

5. One might suspect the chemical stability of the C.47 hemiketal group, particularly under acidic conditions. Indeed, we observed the smooth spiroketalization involving the C.43 and C.51 hydoxy groups. We hoped that this difficulty might be overcome via destabilization of the possible intermediate by introducing the electron-withdrawing group at the C.46 hydroxy group. An acyl group such as $\mathrm{Bz}$ and $\mathrm{Ac}$ met extremely well with our demands. The C.47 methoxy group now became quite stable even under acidic conditions. The deprotection of this group was easily achieved by hydrolyzing first the $\mathrm{C.46}$ acyl group, followed by brief treatment with dilute aqueous acetic acid. The remaining three acyl groups were chosen because of the convenience of synthesis.

The right half of palytoxin was assembled by using two key coupling reactions. First, the C.98-C.99 olefinic bond was stereoselectively introduced by a Wittig reaction. The problem encountered in this coupling reaction was the C.97 epimerization via a ring-opening/closing process (ref. 16). Fortunately, we were able to define specific conditions to achieve the olefination in a good yield without C.97 epimerization. Interestingly, the C.97 epimerization was also observed even in the isolation and purification process of the phosphonium salt. This technical difficulty was overcome by using TSK G3000S polystyrene gel. Second, the C.84-C.85 olefinic bond was introduced by a 
$\mathrm{Ni}(\mathrm{II}) / \mathrm{Cr}$ (II)-mediated coupling. It is worthwhile to note that the coupling was performed by using one equivalent of the cis-iodoolefin in over $95 \%$ yield and also that PDC must be recrystallized from distilled water to perform the oxidation cleanly and efficiently. It is intriguing to point out that the Wittig olefination was extremely sluggish in THF whereas facile in a mixture of hexanes and THF.

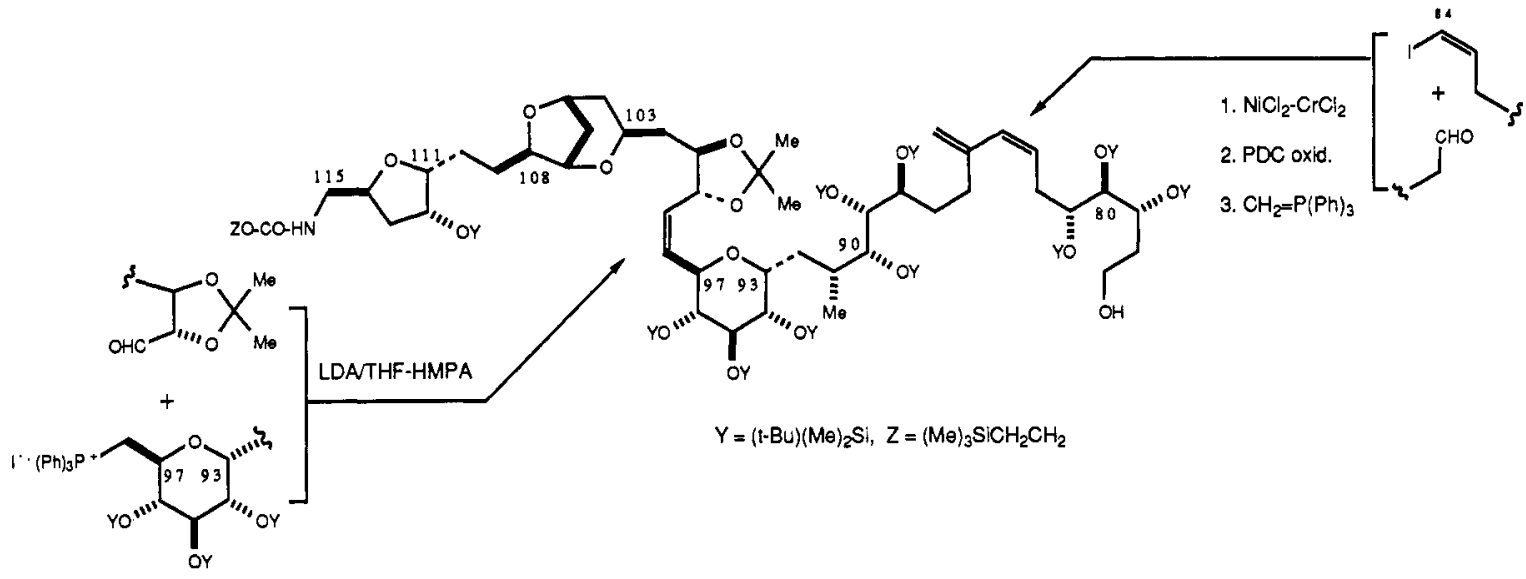

Let us turn our attention to the C.75-C.76 bond formation. As discussed before, we have chosen to use several different protecting groups. There were specific reasons for the choice of each of them. We believed that there was a good chance to deprotect successfully all of them after the entire carbon skeleton was assembled. Nonetheless, we felt it would be safer to utilize a final coupling reaction, which could be performed in organic as well as aqueous media. By this strategy, even if the required deprotections are not practical at the final stage, we will have an alternative option, i.e. to complete the total synthesis by coupling the deprotected forms of the two halves in aqueous solution - note that palytoxin is soluble in water and a mixture of water with certain organic solvents but insoluble in ordinary organic solvents.

Along this analysis, we did a literature search for a coupling reaction, which could be performed in organic as well as aqueous media and noted that the $\mathrm{Pd}(0)$-catalyzed diene synthesis developed by Professor Suzuki and co-workers (ref. 17) seemed to meet ideally our requirement, i.e. a stereospecific diene synthesis possibly in organic as well as aqueous media. Indeed, by using a model compound, we could show that the desired cis,trans-diene was stereospecifically formed in fairly good yield in THF as well as in aqueous THF. However, with the increase in the molecular size of substrates, a sharp decrease in chemical yield was observed. Even for some cases no desired diene formation was detected, clearly suggesting that an improvement should be made in order to apply this process successfully to our work.

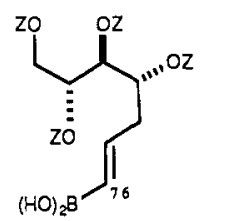

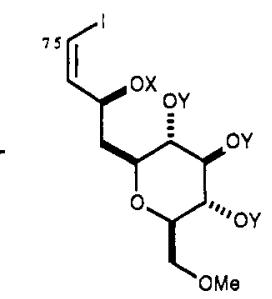

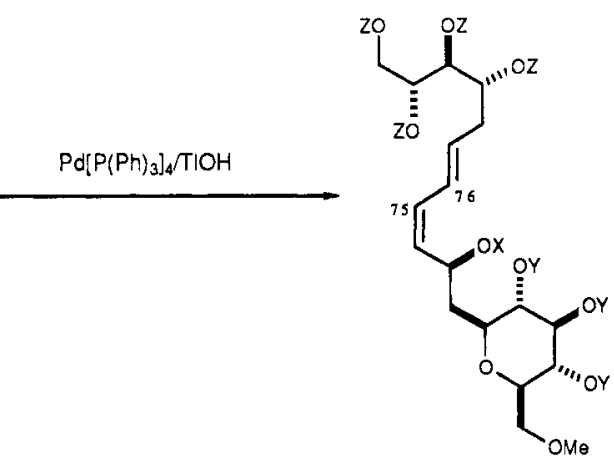

$X=A C, M P M$, or TBS, $Y=A C, M P M$, or TBS, $Z=$ TBS

Professor Suzuki suggested a catalytic cycle with the four critical steps (ref. 17). Among these four steps, the first step, i.e. oxidative addition of vinyliodide to $\operatorname{Pd}(0)$, did not appear to be ratedetermining. This might imply a possibility that by-products were, at least to some extent, formed through the accumulated activated-form of iodoolefins. Thus, if one can make this step ratedetermining for the process, this problem should be eliminated. We were not sure which of the remaining critical steps would be slowest. However, we thought that the rate of the second step, i.e. ligand exchange, should be affected by using different bases which form an insoluble iodide salt in the reaction medium.

This attempt resulted in a dramatic success. Among several bases tested, thallium hydroxide was the best by far. The approximate relative rate was $\mathrm{TlOH}(1,000), \mathrm{Ag}_{2} \mathrm{O}(30)$, and $\mathrm{KOH}(1)$. The reaction rate depended delicately on the C.73 protecting group, i.e. Ac $(<30 \mathrm{sec})$, MPM $(<30 \mathrm{sec})$, and TBS $(60 \mathrm{~min})$. Thus, it became clear that a TBS group was not a suitable protecting group for the C.73 hydoxy group. 
However, this particular substrate gave us an interesting insight into the new reaction conditions. Namely, as the coupling reaction with this substrate was slow enough to follow the rate of disappearance of iodoolefin as well as of appearance of diene, it became evident that the oxidative addition of iodoolefin was the rate-determining step.

From the preparative points of view, the dramatic rate-enhancement realized will result not only in an enormous economy of time and catalyst but also several important consequences. For example, the coupling can now be achieved almost instantaneously even at $0^{\circ} \mathrm{C}$, allowing its application to substrates with fragile functionalities as well as with large molecular weights. In addition, as the oxidative addition step is rate-determining, by-product formation is cleanly eliminated even under stoichiometric conditions.

Before turning our attention to the final coupling reaction, two additional comments on the C.73 functional group should be in order. First, as discussed before, one of the reasons why we chose to apply the Suzuki diene synthesis to our project was the possibility of performing the coupling in an aqueous medium. In this connection, we attempted the coupling reaction using the iodoolefin bearing a free hydroxy group at the C.73 position without any success. It is interesting to note that the corresponding trans-iodoolefin did gave the desired product. Although these experiments suggest an interesting mechanistic insight for the Suzuki diene synthesis, it became clear that a protecting group was required at least for the C.73 hydroxy group. Second, under the DDQ-deprotection conditions, the MPM-protected cis,trans-dienol functionality around the C.73 position was, smoothly and cleanly, oxidized to the corresponding cis,trans-dienone. This transformation seems to have an interesting synthetic potential but, as far as the present synthesis goes, it became obvious that we needed to choose a group other than MPM and TBS as the C.73 protecting group.

Vinylboronic acids required for the Suzuki diene synthesis are usually available via catechol hydroboration of acetylenes. However, we observed that catecholborane attacked the C.115 urethane before the terminal acetylene. Fortunately, this problem was solved by using the reagent introduced by Professor Matteson (ref. 18). This reagent was recommended for homologation of aldehydes but our interest was its intermediate. Because of the diastereotopic nature of the two substituents on the carbanion carbon, one might anticipate the preferential formation of the trans-vinylboronate in the initial condensation step. Indeed, the stereoselectivity experimentally observed ranged $8 \sim 10$ to 1 , favoring the desired trans-vinylboronate, which was hydrolyzed to the trans-vinylboronic acid with brief acid treatment. The trans-vinylboronic acid thus obtained was used for the coupling reaction without purification.

The crucial coupling reaction was performed under the conditions previously established. Using a $1: 1$ molar ratio of the two halves, the desired product was isolated in $70 \%$ overall yield from the primary alcohol. Using a standard procedure, the methyl ester was then converted into the corresponding ketophosphonate almost quantitatively (ref. 19).

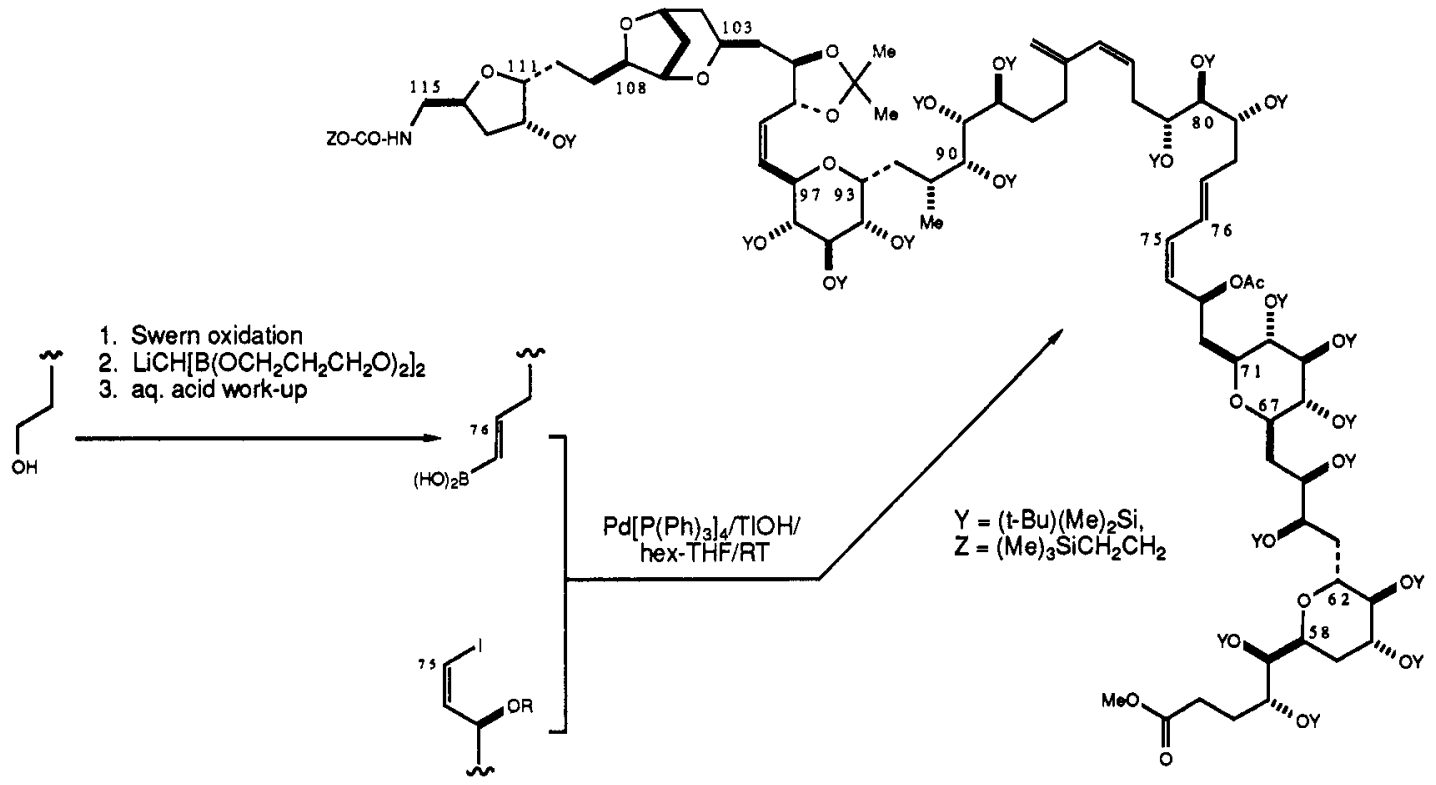

Let us turn our attention to the C.51-C.52 bond formation. Again using suitable model compounds, we have evaluated the feasibility and applicability of various olefin-forming reactions to this case. Among them, the ketophosphonate route yielded the most satisfactory results. Thus, the aldehyde, obtained by oxidation of the left half with $\mathrm{RuCl}_{2}\left[\mathrm{P}(\mathrm{Ph})_{3}\right] 3$ (ref. 20), was coupled with the anion, generated from the ketophosphonate, to yield the desired $\alpha, \beta$-unsaturated ketone in $80 \sim 90 \%$ yield. As one might be concerned, the starting material as well as the product showed interesting but 
undesired reactivities, particularly against basic reagents. These undesired side-reactions were avoided by generating the anion from 1.5 equivalents of ketophosphonate and 1.3 equivalents of $\mathrm{NaH}$ in THF, followed by coupling with 1.0 equivalent of aldehyde. The potential drawback of this procedure was that the reaction was performed at the expense of one half equivalent of the ketophosphonate. Fortunately, however, the excess ketophosphonate could easily be recovered almost quantitatively by silica gel chromatography.

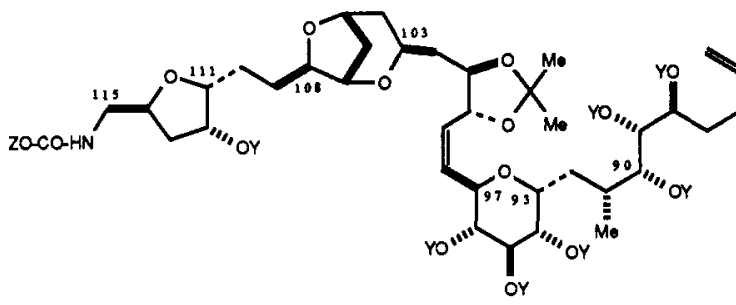<smiles>CCC(O)C(C)CO</smiles>

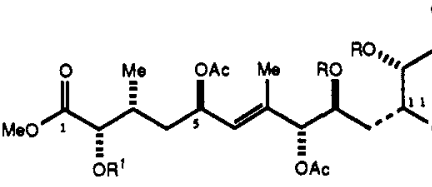<smiles>CC(O)C(C)O</smiles>

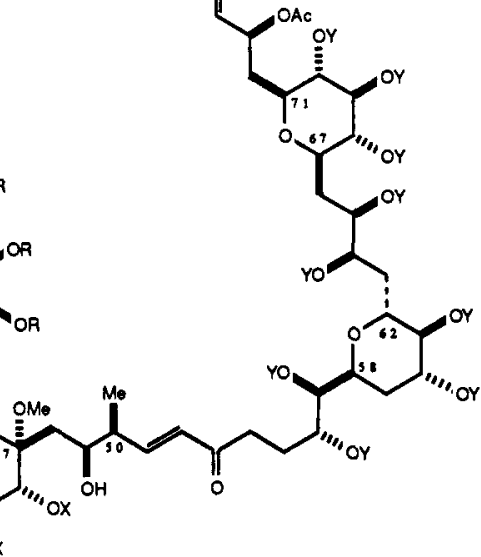

$\mathrm{R}=4-\mathrm{MeOPhCH}_{2}, \mathrm{R}^{1}=2,4-(\mathrm{M} \ominus \mathrm{O})_{2} \mathrm{PhCH}_{2}, \mathrm{X}=\mathrm{Bz}, \mathrm{Y}=(\mathrm{t}-\mathrm{Bu})(\mathrm{Me})_{2} \mathrm{Si}, \mathrm{Z}=(\mathrm{Me})_{3} \mathrm{SiCH}_{2} \mathrm{CH}_{2}$

Needless to point out, the entire carbon skeleton could be assembled by using the inverse order of the two crucial bond-forming reactions, i.e. the C.51-C.52 bond formation followed by the C.75-C.76 bond formation. With respect to the overall efficiency, the first route had the clear edge over the second.

It came time to investigate the method to reduce the C.53 ketone selectively to the desired alcohol. Although the substance with the entire carbon backbone was available in a fair amount, we thought that the studies might be more productive with a suitable model compound. There are two fundamentally different approaches for solving this type of problem; one depends on the substrate structure while the other on the reagent structure. We have tested both possibilities by using the model compound shown. Not surprisingly, sodium borohydride reduction yielded approximately a $1: 1$ mixture of the diastereomeric allylic alcohols. Asymmetric reductions using borohydride- and borane-based reagents did not give promising results but lithium borohydride in the presence of a lanthanide salt produced the desired allylic alcohol as the major product. Among the lanthanide salts tested, $\mathrm{EuCl}_{3}$ gave the best (8:1) ratio.

Stereochemistry Assignment

$$
1 . \mathrm{O}_{3}
$$

2. $\mathrm{NaBH}_{4}$

3. comparison with the authentic samples

Reduction

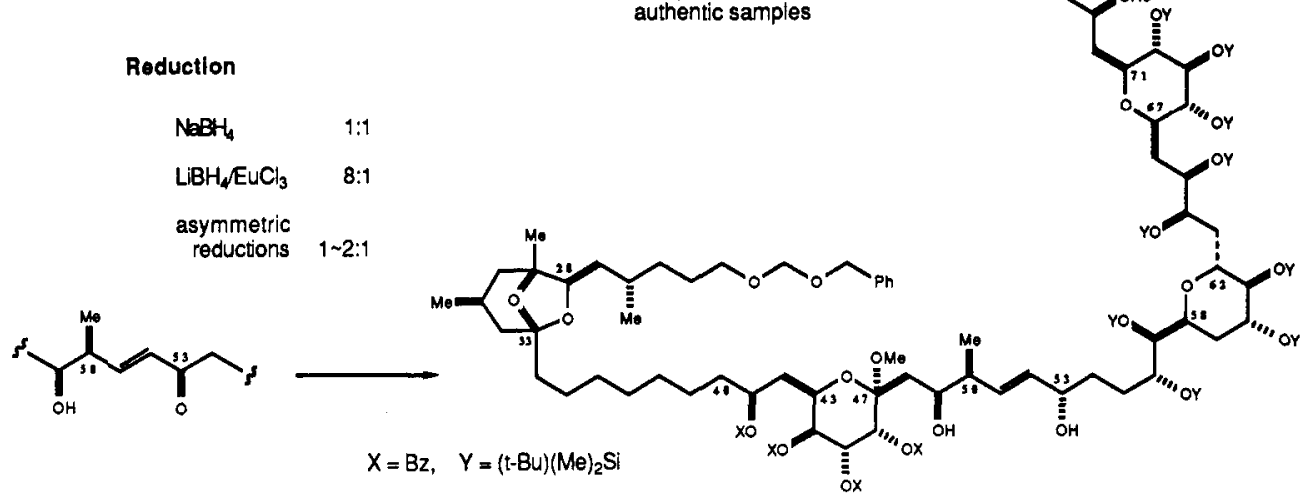


Up to this point, we have not described the details of how the structure of synthetic intermediates was established throughout this synthesis. We have routinely depended on degradation work in addition to the techniques commonly utilized for structure elucidation in organic chemistry. For example, the C.53 stereochemistry of the major product obtained was concluded by subjecting it to ozonization and reduction, to yield the triol of the C.52-C.74 segment, which was then compared with the authentic sample available in our laboratories from the structural study of palytoxin (ref. 3 ).

The lithium borohydride/lanthanide reduction was applied to the $\alpha, \beta$-unsaturated ketone of the real series, and the product was isolated after acetylation. The structure of the long-awaited protected form of palytoxin carboxylic acid was carefully established on the basis of spectroscopic and degradation studies.

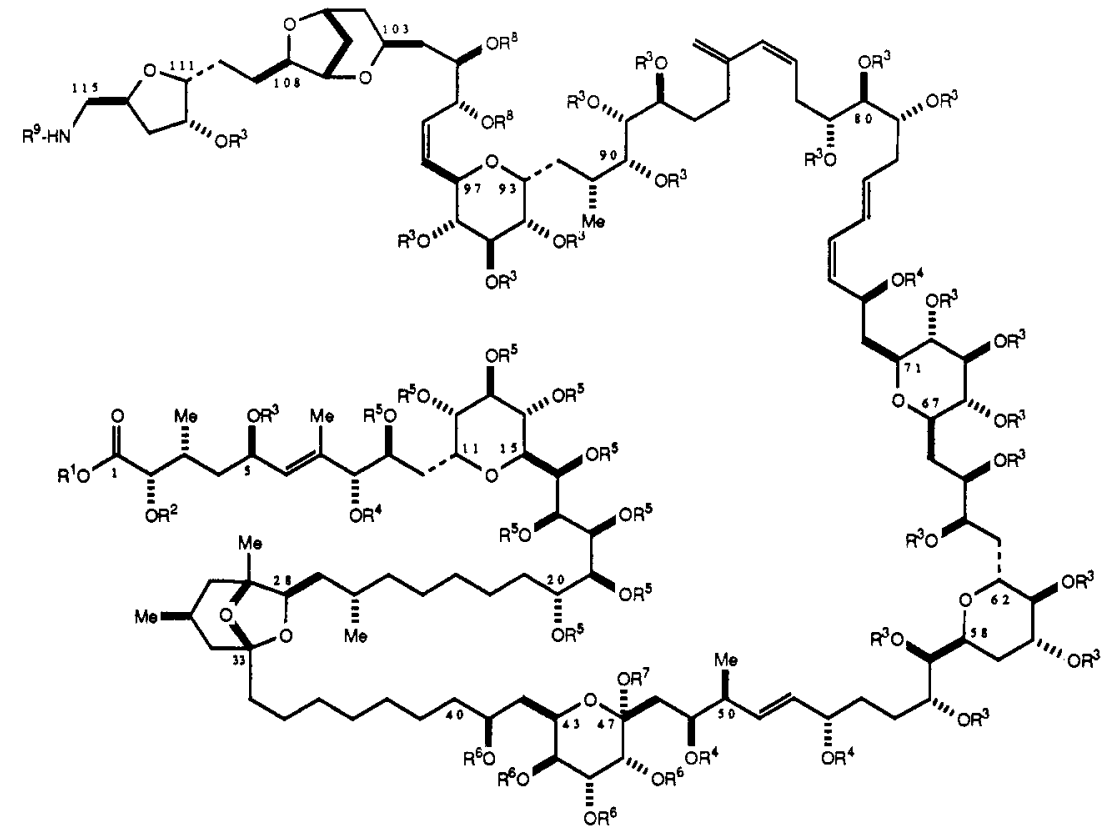

1. DDQ/RT

2. aq. $\mathrm{HClO}_{4} / 25^{\circ} \mathrm{C}$

3. aq. $\mathrm{LIOH} / 25^{\circ} \mathrm{C}$

4. (n-Bu) ${ }_{4} \mathrm{NF} / \mathrm{RT}$

5. aq. AcOH/RT

$R^{1}=\mathrm{Me}, \mathrm{R}^{2}=2,4-(\mathrm{MeO})_{2} \mathrm{PhCH}_{2}, R^{3}=(\mathrm{t}-\mathrm{Bu})(\mathrm{Me})_{2} \mathrm{Si}, R^{4}=\mathrm{Ac}, \mathrm{R}^{5}=4-\mathrm{MeOPhCH}_{2}$, $R^{6}=B z, R^{7}=\mathrm{Me}, R^{8}=$ acetonide, $R^{9}=(\mathrm{Me})_{3} \mathrm{SiCH}_{2} \mathrm{CH}_{2} \mathrm{OCO}$

We were now ready to address the question of deprotection. As discussed before, we anticipated that all the protecting groups should be removed by five synthetic operations, i.e. 1. DDQ treatment to remove the MPM and DMP groups, 2. acid treatment to hydrolyze the acetonide group, 3. base treatment to hydrolyze the acetate and benzoate groups, 4. fluoride treatment to remove the TBS and urethane groups, and 5. aqueous acid treatment to hydrolyze the hemiketal group. The question was then in which order. There were specific reasons why we thought the specific order described above was the best. These include 1. the DDQ treatment must be before the base treatment, 2 . the base treatment must be before the hydrolysis of the C.47 hemiketal, and so on.

The deprotection was carried out under carefully controlled conditions, and the product was isolated in approximately $30 \%$ overall yield by using a TSK G3000S polystyrene gel column. Spectroscopic data, including $2 \mathrm{D}$ NMR spectra, indicated the structure of this product likely to be the $\delta$-lactone. This was an exciting news for two reasons; first, all the protecting groups have successfully been removed and second, the $\delta$-lactone should provide a nice handle to functionalize the C.1 position - note the C.1 functionality of palytoxin. Indeed, we could show the feasibility of such functionalization in the model series. However, we noticed that the reactivity of the $\delta$-lactone in the real series was extremely poor; for example, the conditions of $0.1 \mathrm{M} \mathrm{LiOH}$ at room temperature overnight were not sufficient to hydrolyze completely the $\delta$-lactone. Clearly, we still need to improve the conditions for nucleophilic openings of the $\delta$-lactone. Nonetheless, the completely deprotected carboxylic acid was obtained.

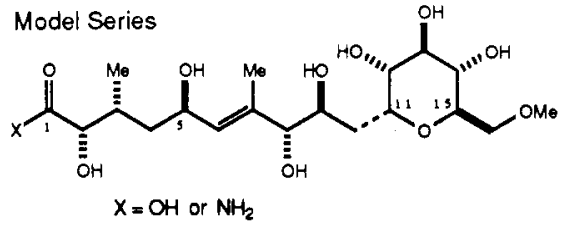

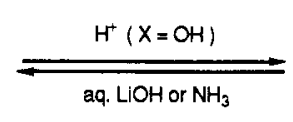

aq. $\mathrm{LiOH}$ or $\mathrm{NH}_{3}$<smiles>COC[C@H]1OC(C[C@H](O)[C@@H](O)/C(C)=C/[C@H]2C[C@H](C)[C@@H](O)C(=O)O2)[C@H](O)[C@@H](O)[C@@H]1O</smiles> 
Here, we would like to mention the recent exciting development in the palytoxin area. Professor Hirata and co-workers continued thorough studies on the natural products existing in the Okinawan Palythoa and succeeded in isolating several minor palytoxins. Among them, the most exciting substance is, at least for us, palytoxin carboxylic acid. The content of this substance in the Okinawan Palythoa is approximately one third of palytoxin and its physiological activity compares well with palytoxin itself (ref. 21). Thus, the compound we have just synthesized tumed out to be one of the naturally occurring palytoxins!

On comparison of biological activity, chromatographic behavior and spectroscopic data, the synthetic substance was indistinguishable from naturally occurring palytoxin carboxylic acid.

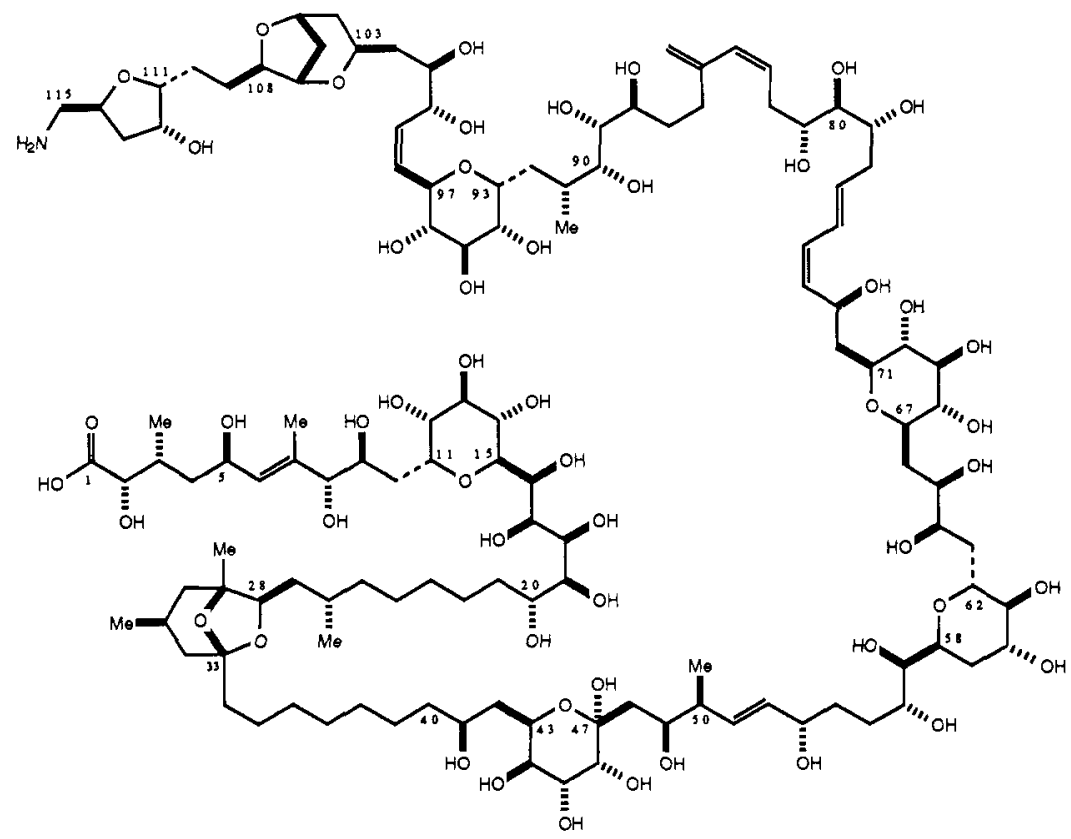

Palytoxin Carboxylic Acid

Acknowledgements I would like to express the warmest appreciation to the co-workers whose spirit, dedication, stamina, and skill have enabled us to bring the stimulating, exciting, challenging, and rewarding venture to a successful conclusion. The co-workers who have energetically and enthusiastically participated in the work outlined are acknowledged in references. Financial assistance from the National Institutes of Health (NS-12108) and the National Science Foundation (CHE $86-105050$ ) is gratefully acknowledged.

\section{REFERENCES}

1. For reviews on palytoxin, see: R. E. Moore, "Prog. Chem. Org. Nat. Prod.", Vol. 48, page 81 ff, Springer-Verlag, New York, 1985 and reviews cited therein.

2. For the gross structure of palytoxin, see: (a) D. Uemura, K. Ueda, Y. Hirata, H. Naoki, and T. Iwashita, Tetrahedron Lett., 22, 2781 (1981) and references cited therein. (b) R. E. Moore and G. Bartolini, J.Am. Chem. Soc. 103, 2491 (1981) and references cited therein.

3. For the stereochemistry assignment primarily based on organic synthesis, see: J. K. Cha, W. J. Christ, J. M. Finan, H. Fujioka, Y. Kishi, L. L. Klein, S. S. Ko, J. Leder, W. W. McWhorter, Jr., K.-P. Pfaff, M. Yonaga, D. Uemura, and Y. Hirata, J. Am Chem. Soc., 104, 7369 (1982) and preceding papers. (b) For the stereochemistry assignment primarily based on spectroscopic methods, see: R. E. Moore, G. Bartolini, J. Barchi, A. A. Bothner-By, J. Dadok, and J. Ford, J. Am. Chem. Soc., 104, 3776 (1982).

4. S. A. Babirad, Y. Wang, P. G. Goekjian, and Y. Kishi, J. Org. Chem., 52, 4825 (1987). 
5. R. U. Lemieux, A. A. Pavia, J. C. Martin, and K. A. Watanabe, Can. J. Chem., 47, 4427 (1969). Also see, R. U. Lemieux and S. Kato, Tetrahedron, 30, 1933 (1974).

6. There are many excellent reviews on conformational analysis of saccharides. For example, see: K. Boch, Pure Appl. Chem, 55, 605 (1983) and 59, 1447 (1987) and reviews cited therein.

7. P. G. Goekjian, Y. Kishi, W. H. Miller, D. M. Ryckman, and Y. Wang, unpublished results.

8. K.-H. Budt, J. Goudar, Y. Kishi, T. Li, and J.-M. Vatele, unpublished results.

9. For synthetic studies on palytoxin from our laboratories, see: Y. Kishi, Chemica Scripta, 27, 573 (1987) and references cited therein. Also see see references $14 \mathrm{a}$ and 19.

10. We were concerned primarily with the syntheses of building blocks for the period of 1982-1985; Drs. J.-M. Beau, S. H. Cheon, W. J. Christ, L. D. Hawkins, S. H. Kang, A. E. Stutz, M. Taniguchi, K. Ueda, J. B. White, M. Yonaga, and M. Yoshikawa were engaged on the project in this period.

11. We have been concerned primarily with the coupling reactions for the period of 1985-present; Drs. R. W. Armstrong, W. J. Chrsit, W.-H. Ham, H. Jin, M. J. Martenelli, M. Nakata, F. X. Talamas, J. A. Tino, K. Ueda, and J. Uenishi have been engaged on the project in this period.

12. K. Takai, K. Kimura, T. Kuroda, T. Hiyama, and H. Nozaki, Tetrahedron Lett., 24, 5281 (1983).

13. Y. Okude, S. Hirano, T. Hiyama, and H. Nozaki, I. Am. Chem. Soc, 29, 3179 (1977).

14. (a) H. Jin, J. Uenishi, W. J. Christ, and Y. Kishi, I. Am. Chem. Soc., 108, 5644 (1986). (b) K. Takai, M. Tagashira, T. Kuroda, T. Oshima, K. Uchimoto, and H. Nozaki, L. Am. Chem. Soc. 108, 6048 (1986)

15. Y. Oikawa, T. Yoshioka, and O. Yonemitsu, Tetrahedron Lett, 23, 885 and 889 (1982).

16. This problem is known in the literature. See: J. A. Secrist, III and K. D. Barnes, J. Org. Chem., 45, 4526 (1980) and references cited therein.

17. N. Miyaura, K. Yamada, H. Suginome, and A. Suzuki, L.Am. Chem. Soc., 107, 972 (1985) and references cited therein.

18. D. S. Matteson and R. J. Moody, Organometallics, 1, 20 (1982).

19. J. Uenishi, J.-M. Beau, R. W. Armstrong, and Y. Kishi, J. Am. Chem. Soc, 109, 4756 (1987).

20. H. Tomioka, K. Takai, K. Oshima, and H. Nozaki, Tetrahedron Lett, 22, 1605 (1981).

21. (a) D. Uemura, T. Iwashita, H. Naoki, and Y. Hirata, Annual Meeting of The Chemical Society of Japan, April (1986). (b) D. Uemura, Y. Hirata, T. Iwashita, and H. Naoki, Tetrahedron, 41, 1007 (1985).

22. We are indebted to Professors Hirata and Uemura for a sample of natural palytoxin carboxylic acid and palytoxin. 